\title{
Dynamic Contact Problem for Viscoelastic von Kármán-Donnell Shells
}

\author{
Igor Bock ${ }^{1}$ and Jiří Jarušek ${ }^{2}$ \\ 1 Institute of Computer Science and Mathematics FEI, \\ Slovak University of Technology, 81219 Bratislava 1, Slovakia \\ igor.bock@stuba.sk \\ 2 Institute of Mathematics, Academy of Sciences of the Czech Republic, \\ Žitná 25, 11567 Praha 1, Czech Republic \\ jarusek@math.cas.cz
}

\begin{abstract}
We deal with initial-boundary value problems describing vertical vibrations of viscoelastic von Kármán-Donnell shells with a rigid inner obstacle. The short memory (Kelvin-Voigt) material is considered. A weak formulation of the problem is in the form of the hyperbolic variational inequality. We solve the problem using the penalization method.
\end{abstract}

Keywords: Von Kármán-Donnell shell, unilateral dynamic contact, viscoelasticity, solvability, penalty approximation.

\section{Introduction}

Contact problems represent an important but complex topic of applied mathematics. Its complexity profounds if the dynamic character of the problem is respected. For elastic problems there is only a very limited amount of results available (cf. 3] and there cited literature). Viscosity makes possible to prove the existence of solutions for a broader set of problems for membranes, bodies as well as for linear models of plates. The presented results extend the research made in [2, where the problem for a viscoelastic short memory von Kármán plate in a dynamic contact with a rigid obstacle was considered. Our results also extend the research made for the quasistatic contact problems for viscoelastic shells (cf. [1]). A thin isotropic shallow shell occupies the domain

$$
G=\left\{(x, z) \in R^{3}: x=\left(x_{1}, x_{2}\right) \in \Omega,|z-\mathcal{Z}|<h / 2\right\},
$$

where $h>0$ is the thickness of the shell, $\Omega \subset \mathbb{R}^{2}$ is a bounded simply connected domain in $\mathbb{R}$ with a sufficiently smooth boundary $\Gamma$. We set $I \equiv(0, T)$ a bounded time interval, $Q=I \times \Omega, S=I \times \Gamma$. The unit outer normal vector is denoted by $\mathbf{n}=\left(n_{1}, n_{2}\right), \tau=\left(-n_{2}, n_{1}\right)$ is the unit tangent vector. The displacement is denoted by $\mathbf{u} \equiv\left(u_{i}\right)$. The strain tensor is defined as

$$
\varepsilon_{i j}(\mathbf{u})=\frac{1}{2}\left(\partial_{i} u_{j}+\partial_{j} u_{i}+\partial_{i} u_{3} \partial_{j} u_{3}\right)-k_{i j} u_{3}-x_{3} \partial_{i j} u_{3}, i, j=1,2
$$

D. Hömberg and F. Tröltzsch (Eds.): CSMO 2011, IFIP AICT 391, pp. 70-75, 2013.

(C) IFIP International Federation for Information Processing 2013 
with $k_{12}=k_{21}=0$ and the curvatures $k_{i i}>0, i=1,2$.

Further, we set

$$
[u, v] \equiv \partial_{11} u \partial_{22} v+\partial_{22} u \partial_{11} v-2 \partial_{12} u \partial_{12} v
$$

In the sequel, we denote by $W_{p}^{k}(M), k \geq 0, p \in[1, \infty]$ the Sobolev spaces defined on a domain or an appropriate manifold $M$. By $\stackrel{\circ}{W}_{p}^{k}(M)$ the spaces with zero traces are denoted. If $p=2$ we use the notation $H^{k}(M), \stackrel{\circ}{H}^{k}(M)$. The duals to $\stackrel{\circ}{H}^{k}(M)$ are denoted by $H^{-k}(M)$. For the anisotropic spaces $W_{p}^{k}(M), k=$ $\left(k_{1}, k_{2}\right) \in \mathbb{R}_{+}^{2}, \quad k_{1}$ is related with the time variable while $k_{2}$ with the space variables. We shall use also the Bochner-type spaces $W_{p}^{k}(I ; X)$ for a time interval $I$ and a Banach space $X$. Let us remark that for $k \in(0,1)$ their norm is defined by the relation

$$
\|w\|_{W_{p}^{k}(I ; X)}^{p} \equiv \int_{I}\|w(t)\|_{X}^{p} d t+\int_{I} \int_{I} \frac{\|w(t)-w(s)\|_{X}^{p}}{|s-t|^{1+k p}} d s d t .
$$

By $C(M)$ we denote the spaces of continuous functions on a (possibly relatively) compact manifold $M$. They are equipped with the max-norm. Analogously the spaces $C(M ; X)$, are introduced for a Banach space $X$. The following generalization of the Aubin's compactness lemma verified in [4] Theorem 3.1 will be essentially used:

Lemma 1. Let $B_{0} \hookrightarrow \hookrightarrow B \hookrightarrow B_{1}$ be Banach spaces, the first reflexive and separable. Let $1<p<\infty, 1 \leq r<\infty$. Then

$$
W \equiv\left\{v ; v \in L_{p}\left(I ; B_{0}\right), \dot{v} \in L_{r}\left(I, B_{1}\right)\right\} \hookrightarrow \hookrightarrow L_{p}(I ; B) .
$$

\section{Short Memory Material}

\subsection{Problem Formulation}

Employing the Einstein summation, the constitutional law has the form

$\sigma_{i j}(\mathbf{u})=\frac{E_{1}}{1-\mu^{2}} \partial_{t}\left((1-\mu) \varepsilon_{i j}(\mathbf{u})+\mu \delta_{i j} \varepsilon_{k k}(\mathbf{u})\right)+\frac{E_{0}}{1-\mu^{2}}\left((1-\mu) \varepsilon_{i j}(\mathbf{u})+\mu \delta_{i j} \varepsilon_{k k}(\mathbf{u})\right)$.

The constants $E_{0}, E_{1}>0$ are the Young modulus of elasticity and the modulus of viscosity, respectively. We shall use the abbreviation $b=h^{2} /\left(12 \varrho\left(1-\mu^{2}\right)\right)$, where $h>0$ is the shell thickness and $\varrho$ is the density of the material. We involve the rotation inertia expressed by the term $a \Delta \ddot{u}$ in the first equation of the considered system with $a=\frac{h^{2}}{12}$. It will play the crucial role in the deriving a strong convergence of the sequence of velocities $\left\{\dot{u}_{m}\right\}$ in the appropriate space. We assume the shell clamped on the boundary. We generalize the dynamic elastic model due to the von Kármán-Donnell theory mentioned in [6]. The classical 
formulation for the deflection $u_{3} \equiv u$ and the Airy stress function $v$ is then the initial-value problem

$$
\begin{gathered}
\ddot{u}+a \Delta \ddot{u}+b\left(E_{1} \Delta^{2} \dot{u}+E_{0} \Delta^{2} u\right)-[u, v]-\Delta_{k} * v=f+g, \\
u-\Psi \geq 0, g \geq 0,(u-\Psi) g=0 \\
\begin{array}{c}
\Delta^{2} v+E_{1} \partial_{t}\left(\frac{1}{2}[u, u]+k_{11} \partial_{22} u+k_{22} \partial_{11} u\right) \\
+E_{0}\left(\frac{1}{2}[u, u]+\Delta_{k} u\right)=0
\end{array} \\
u=\partial_{n} u=v=\partial_{n} v=0 \text { on } S \\
u(0, \cdot)=u_{0}, \dot{u}(0, \cdot)=u_{1} \text { on } \Omega .
\end{gathered}
$$

The obstacle function $\Psi \in L_{\infty}(\Omega)$ is fulfilling $0<U_{0} \leq u_{0}-\Psi$ in $\Omega$ and

$$
\begin{aligned}
& \Delta_{k} u \equiv \partial_{11}\left(k_{22} u\right)+\partial_{22}\left(k_{11} u\right), \\
& \Delta_{k}^{*} v \equiv k_{22} \partial_{11} v+k_{11} \partial_{22} v .
\end{aligned}
$$

We define the operators $L: H^{2}(\Omega) \rightarrow \stackrel{\circ}{H}^{2}(\Omega), \Phi: H^{2}(\Omega) \times H^{2}(\Omega) \rightarrow \stackrel{\circ}{H}^{2}(\Omega)$ by uniquely solved equations

$$
\begin{aligned}
(\Delta L u, \Delta w) & \equiv\left(\Delta_{k} u, w\right) \forall w \in \stackrel{\circ}{H}^{2}(\Omega), \\
(\Delta \Phi(u, v), \Delta w) & \equiv([u, v], w) \forall w \in \stackrel{\circ}{H}^{2}(\Omega) .
\end{aligned}
$$

with the inner product $(\cdot, \cdot)$ in the space $L_{2}(\Omega)$. The operator $L$ is linear and compact. The bilinear operator $\Phi$ is symmetric and compact. Moreover due to Lemma 1 from [5] $\Phi: H^{2}(\Omega)^{2} \rightarrow W_{p}^{2}(\Omega), 2<p<\infty$ and

$$
\|\Phi(u, v)\|_{W_{p}^{2}(\Omega)} \leq c\|u\|_{H^{2}(\Omega)}\|v\|_{W_{p}^{1}(\Omega)} \forall u \in H^{2}(\Omega), v \in W_{p}^{1}(\Omega) .
$$

We have also $L: H^{2}(\Omega) \mapsto W_{p}^{2}(\Omega), \quad 2<p<\infty$ and

$$
\|L u\|_{W_{p}^{2}(\Omega)} \leq c\|u\|_{H^{2}(\Omega)} \forall u \in H^{2}(\Omega) .
$$

For $u, y \in L_{2}\left(I ; H^{2}(\Omega)\right)$ we define the bilinear form $A$ by

$$
A(u, y):=b\left(\partial_{k k} u \partial_{k k} y+\mu\left(\partial_{11} u \partial_{22} y+\partial_{22} u \partial_{11} y\right)+2(1-\mu) \partial_{12} u \partial_{12} y\right) \text {. }
$$

We introduce shifted cone $\mathcal{K}$ by

$$
\mathcal{K}:=\left\{y \in H^{1,2}(Q) ; \dot{y} \in L_{2}\left(I, \stackrel{\circ}{H}^{1}(\Omega) ; y \geq \Psi\right\} .\right.
$$

Then the variational formulation of the problem (1+3) has the form of

Problem $\mathcal{P}$. Find $u \in \mathcal{K}$ such that $\dot{u} \in L_{2}\left(I ; \stackrel{\circ}{H}^{2}(\Omega)\right)$ and

$$
\begin{aligned}
& \int_{Q}\left(E_{1} A(\dot{u}, y-u)+E_{0} A(u, y-u)\right) d x d t \\
+ & \int_{Q}\left[u, E_{1} \partial_{t}\left(\frac{1}{2} \Phi(u, u)+L u\right)+E_{0}\left(\frac{1}{2} \Phi(u, u)+L u\right)\right](y-u) d x d t \\
+ & \int_{Q} \Delta_{k}\left(E_{1} \partial_{t}\left(\frac{1}{2} \Phi(u, u)+L u\right)+E_{0}\left(\frac{1}{2} \Phi(u, u)+L u\right)\right)(y-u) d x d t \\
- & \int_{Q}(a \nabla \dot{u} \cdot \nabla(\dot{y}-\dot{u})+\dot{u}(\dot{y}-\dot{u})) d x d t \\
+ & \int_{\Omega}(a \nabla \dot{u} \cdot \nabla(y-u)+\dot{u}(y-u))(T, \cdot) d x \\
\geq & \int_{\Omega}\left(a \nabla u_{1} \cdot \nabla\left(y(0, \cdot)-u_{0}\right)+u_{1}\left(y(0, \cdot)-u_{0}\right)\right) d x \\
+ & \int_{Q} f\left(y_{1}-u\right) d x d t \forall y \in \mathcal{K} .
\end{aligned}
$$




\subsection{The Penalization}

For any $\eta>0$ we define the penalized problem

Problem $\mathcal{P}_{\eta}$. Find $u \in H^{1,2}(Q)$ such that $\dot{u} \in L_{2}\left(I ; \stackrel{\circ}{H}^{2}(\Omega)\right), \ddot{u} \in L_{2}\left(I ; \stackrel{\circ}{H}^{1}(\Omega)\right)$,

$$
\begin{aligned}
& \int_{Q}\left(\ddot{u} z+a \nabla \ddot{u} \cdot \nabla z+E_{1} A(\dot{u}, z)+E_{0} A(u, z)\right) d x d t \\
+ & \int_{Q}\left[u, E_{1} \partial_{t}\left(\frac{1}{2} \Phi(u, u)+L u\right)+E_{0}\left(\frac{1}{2} \Phi(u, u)+L u\right)\right] z d x d t \\
+ & \int_{Q} \Delta_{k}\left(E_{1} \partial_{t}\left(\frac{1}{2} \Phi(u, u)+L u\right)+E_{0}\left(\frac{1}{2} \Phi(u, u)+L u\right)\right) z d x d t \\
= & \int_{Q}\left(f+\eta^{-1}(u-\Psi)^{-}\right) z d x d t \forall z \in L_{2}\left(I ; H^{2}(\Omega)\right)
\end{aligned}
$$

and the conditions (3) remain valid.

Lemma 2. Let $f \in L_{2}(Q), u_{0} \in \stackrel{\circ}{H}^{2}(\Omega)$, and $u_{1} \in \stackrel{\circ}{H}^{1}(\Omega)$. Then there exists a solution $u$ of the problem $\mathcal{P}_{\eta}$.

Proof. Let us denote by $\left\{w_{i} \in \stackrel{\circ}{H}^{2}(\Omega) ; i=1,2, \ldots\right\}$ a basis of $\stackrel{\circ}{H}^{2}(\Omega)$ orthonormal in $H^{1}(\Omega)$ with respect to the inner product

$$
(u, v)_{a}=\int_{\Omega}(u v+a \nabla u \cdot \nabla v) d x, u, v \in H^{1}(\Omega) .
$$

We construct the Galerkin approximation $u_{m}$ of a solution in a form

$$
\begin{gathered}
u_{m}(t)=\sum_{i=1}^{m} \alpha_{i}(t) w_{i}, \alpha_{i}(t) \in \mathbb{R}, i=1, \ldots, m, m \in N, \\
\left(\ddot{u}_{m}(t), w_{i}\right)_{a}+\int_{\Omega}\left(E_{1} A\left(\dot{u}_{m}(t), w_{i}\right)+E_{0} A\left(u_{m}(t), w_{i}\right)\right) d x+ \\
\int_{\Omega} \Delta\left(E_{1} \partial_{t}\left(\frac{1}{2} \Phi\left(u_{m}, u_{m}\right)+L u_{m}\right)+E_{0}\left(\frac{1}{2} \Phi\left(u_{m}, u_{m}\right)+L u_{m}\right)\right) \\
\times \Delta\left(\Phi\left(u_{m}, w_{i}\right)+L w_{i}\right) d x \\
=\int_{\Omega}\left(f(t)+\eta^{-1}\left(u_{m}(t)-\Psi\right)^{-}\right) w_{i} d x, i=1, \ldots, m, \\
u_{m}(0)=u_{0 m}, \dot{u}_{m}(0)=u_{1 m}, u_{0 m} \rightarrow u_{0} \text { in } \dot{H}^{2}(\Omega), u_{1 m} \rightarrow u_{1} \text { in } \stackrel{\circ}{H}^{1}(\Omega) .
\end{gathered}
$$

After multiplying the equation (14) by $\dot{\alpha}_{i}(t)$, summing up with respect to $i$, taking in mind the definitions of the operators $\Phi, L$ and integrating we obtain the a priori estimates not depending on $m$ :

$$
\begin{aligned}
& \left\|\dot{u}_{m}\right\|_{L_{2}\left(I ; \dot{H}^{2}(\Omega)\right)}^{2}+\left\|\dot{u}_{m}\right\|_{L_{\infty}\left(I ; \dot{H}^{1}(\Omega)\right)}^{2}+\left\|u_{m}\right\|_{L_{\infty}\left(I ; \dot{H}^{2}(\Omega)\right)}^{2} \\
& +\left\|\partial_{t} \Phi\left(u_{m}, u_{m}\right)\right\|_{L_{2}\left(I ; \dot{H}^{2}(\Omega)\right)}^{2}+\left\|\partial_{t} L u_{m}\right\|_{L_{2}\left(I ; \dot{H}^{2}(\Omega)\right)}^{2} \\
& +\eta^{-1}\left\|\left(u_{m}-\Psi\right)^{-}\right\|_{L_{\infty}\left(I ; L_{2}(\Omega)\right)} \leq c \equiv c\left(f, u_{0}, u_{1}\right) .
\end{aligned}
$$

Moreover the estimates (8), (9) imply

$$
\left\|\partial_{t} \Phi\left(u_{m}, u_{m}\right)\right\|_{L_{2}\left(I ; W_{p}^{2}(\Omega)\right)}+\left\|\partial_{t} L u_{m}\right\|_{L_{2}\left(I ; W_{p}^{2}(\Omega)\right)} \leq c_{p} \forall p>2 .
$$

After multiplying the equation (14) by $\ddot{\alpha}_{i}(t)$, summing up and integrating we obtain the estimate of $\ddot{u}_{m}$

$$
\left\|\ddot{u}_{m}\right\|_{L_{2}\left(I ; H^{1}(\Omega)\right)} \leq c_{\eta}, m \in \mathbb{N} .
$$


Applying the estimates (16)-(18), the compact imbedding theorem and the interpolation, we obtain for any $p \in[1, \infty)$, a subsequence of $\left\{u_{m}\right\}$ (denoted again by $\left.\left\{u_{m}\right\}\right)$, a function $u$ and the convergences

$$
\begin{aligned}
& \ddot{u}_{m} \rightarrow \ddot{u} \text { in } L_{2}\left(I ; H^{1}(\Omega)\right), \\
& \dot{u}_{m} \rightarrow^{*} \dot{u} \text { in } L_{\infty}\left(I ; \stackrel{H}{H}^{1}(\Omega)\right), \\
& \dot{u}_{m} \rightarrow \dot{u} \text { in } L_{2}\left(I ; \stackrel{H}{ }^{2}(\Omega)\right), \\
& \dot{u}_{m} \rightarrow \dot{u} \text { in } L_{p}\left(I ; \stackrel{\circ}{ }^{(}(\Omega)\right) \cap L_{\infty}\left(I ; H^{2-\varepsilon}(\Omega)\right) \forall \varepsilon>0, \\
& u_{m} \rightarrow u \text { in } C\left(\bar{I} ; W_{p}^{1}(\Omega),\right. \\
& \partial_{t}\left(\frac{1}{2} \Phi\left(u_{m}, u_{m}\right)+L u_{m}\right) \rightarrow \partial_{t}\left(\frac{1}{2} \Phi(u, u)+L u\right) \text { in } L_{2}\left(I ; W_{p}^{2}(\Omega)\right)
\end{aligned}
$$

implying that a function $u$ fulfils the identity (12). The initial conditions (3) follow due to (15) and the proof of the existence of a solution is complete.

\subsection{Solving the Original Problem}

We verify the existence theorem

Theorem 1. Let $f \in L_{2}(Q), u_{i} \in \stackrel{\circ}{H}^{2}(\Omega), i=0,1,0<U_{0} \leq u_{0}-\Psi$. Then there exists a solution of the Problem $\mathcal{P}$.

Proof. We perform the limit process for $\eta \rightarrow 0$. We write $u_{\eta}$ for the solution of the problem $\mathcal{P}_{1, \eta}$. The a priori estimates (16) imply the estimates

$$
\begin{aligned}
& \left\|\dot{u}_{\eta}\right\|_{L_{2}\left(I ; \dot{H}^{2}(\Omega)\right)}^{2}+\left\|\dot{u}_{\eta}\right\|_{L_{\infty}\left(I ; \stackrel{H}{1}^{1}(\Omega)\right)}^{2}+\left\|u_{\eta}\right\|_{L_{\infty}\left(I ; \dot{H}^{2}(\Omega)\right)}^{2} \\
& +\left\|\partial_{t} \Phi\left(u_{\eta}, u_{\eta}\right)\right\|_{L_{2}\left(I ; W_{p}^{2}(\Omega)\right)}^{2}+\left\|\partial_{t} L u_{\eta}\right\|_{L_{2}\left(I ; W_{p}^{2}(\Omega)\right)}^{2} \\
& +\eta^{-1}\left\|\left(u_{\eta}-\Psi\right)^{-}\right\|_{L_{\infty}\left(I ; L_{2}(\Omega)\right)} \leq c_{p}, p>2 .
\end{aligned}
$$

To get the crucial estimate for the penalty, we put $z=u_{0}-u_{\eta}(t, \cdot)$ in (12) and obtain the estimate

$$
\begin{gathered}
0 \leq U_{0} \int_{Q} \eta^{-1}\left(u_{\eta}-\Psi\right)^{-} d x d t \leq \int_{Q} \| \eta^{-1}\left(u_{\eta}-\Psi\right)^{-}\left(u_{0}-\Psi\right) d x d t \\
\leq \int_{Q} \| \eta^{-1}\left(u_{\eta}-\Psi\right)^{-}\left(u_{0}-u_{\eta}\right) d x d t \\
=\int_{Q}\left(\dot{u}_{\eta}^{2}+a\left|\nabla \dot{u}_{\eta}\right|^{2}+A\left(\left(E_{1} \partial_{t} u_{\eta}+E_{0} u_{\eta}\right), u_{0}-u_{\eta}\right)\right. \\
+E_{1} \partial_{t}\left(\Delta\left(L u_{\eta}+\frac{1}{2} \Phi\left(u_{\eta}, u_{\eta}\right)\right)\right) \Delta\left(L\left(u_{0}-u_{\eta}\right)+\Phi\left(u_{\eta}, u_{0}-u_{\eta}\right)\right) \\
\left.+E_{0} \Delta\left(L u_{\eta}+\frac{1}{2} \Phi\left(u_{\eta}, u_{\eta}\right)\right) \Delta\left(L\left(u_{0}-u_{\eta}\right)+\Phi\left(u_{\eta}, u_{0}-u_{\eta}\right)\right)\right) d x d t \\
-\int_{Q} f\left(u_{0}-u_{\eta}\right) d x d t+\int_{\Omega}\left(\left(\dot{u}_{\eta}\left(u_{0}-u_{\eta}\right)+a \nabla \dot{u}_{\eta} \cdot \nabla\left(u_{0}-u_{\eta}\right)\right)(T, \cdot)\right) d x .
\end{gathered}
$$

Applying the a priori estimates (20) we obtain

$$
\left\|\eta^{-1} u_{\eta}^{-}\right\|_{L_{1}(Q)} \leq c\left(f, u_{0}, u_{1}, \Psi\right) .
$$

With respect to Dirichlet conditions we obtain from (12) and (21) the dual estimate

$$
\left\|-a \Delta \ddot{u}_{\eta}+\ddot{u}_{\eta}\right\|_{L_{1}\left(I ; H^{-2}(\Omega)\right)} \leq c .
$$


We take the sequence $\left\{u_{k}\right\} \equiv\left\{u_{\eta_{k}}\right\}, \eta_{k} \rightarrow 0+$.

After applying the Lemma 1 with the spaces

$$
B_{0}=L_{2}(\Omega), B=H^{-1}(\Omega), B_{1}=H^{-2}(\Omega)
$$

we obtain the relative compactness of the sequence $\left\{-a \Delta \dot{u}_{k}+\dot{u}_{k}\right\}$ in $L_{2}\left(I ; H^{-1}(\Omega)\right.$ and with the help of the test function $\dot{u}_{k}-\dot{u}$ the crucial strong convergence

$$
\dot{u}_{k} \rightarrow \dot{u} \text { in } L_{2}\left(I ; \stackrel{\circ}{H}^{1}(\Omega)\right) .
$$

Simultaneously we have the convergences

$$
\begin{aligned}
& \dot{u}_{k} \rightarrow \dot{u} \text { in } L_{2}\left(I ; \stackrel{\circ}{ }^{2}(\Omega)\right), \\
& \dot{u}_{k} \rightarrow \dot{u} \text { in } L_{2}\left(I ; W_{p}^{1}(\Omega)\right), \\
& \frac{1}{2} \partial_{t} \Phi\left(u_{k}, u_{k}\right)+\partial_{t} L u_{k} \rightarrow \frac{1}{2} \partial_{t} \Phi(u, u)+\partial_{t} L u \text { in } L_{2}\left(I ; W_{p}^{2}(\Omega)\right) .
\end{aligned}
$$

It can be verified after inserting the test function $z=y-u_{k}$ in (12) for $y \in \mathcal{K}$, performing the integration by parts in the terms containing $\ddot{u}$, applying the convergences (23), (24), using the definitions of the operators $L, \Phi$ in (6), (7) and the weak lower semicontinuity that the limit function $u$ is a solution of the original problem $\mathcal{P}$.

Remark 1. The existence Theorem 1 can be after some modification verified also for another types of boundary conditions.

Acknowledgments. The work presented here was supported by the Czech Academy of Sciences under grant P201/12/0671 and under the Institutional research plan RVD 67985840, by the Czech Ministery of Education under grant MEB 0810045, by the APVV Agency of Slovak Republic under grant 0011-09 and by Ministry of Education of the Slovak Republic under VEGA grant 1/0426/12.

\section{References}

1. Bock, I.: On a pseudoparabolic system for a viscoelastic shallow shell. PAMM Proc. Appl. Math. Mech. 6, 621-622 (2006)

2. Bock, I., Jarušek, J.: Unilateral dynamic contact of viscoelastic von Kármán plates. Advances in Math. Sci. and Appl. 16, 175-187 (2006)

3. Eck, C., Jarušek, J., Krbec, M.: Unilateral Contact Problems in Mechanics. Variational Methods and Existence Theorems. Monographs \& Textbooks in Pure \& Appl. Math., vol. 270. Chapman \& Hall/CRC (Taylor \& Francis Group), Boca Raton, London, New York, Singapore (2005)

4. Jarušek, J., Málek, J., Nečas, J., Šverák, V.: Variational inequality for a viscous drum vibrating in the presence of an obstacle. Rend. Mat., Ser. VII 12, 943-958 (1992)

5. Koch, H., Stachel, A.: Global existence of classical solutions to the dynamical von Kármán equations. Math. Methods in Applied Sciences 16, 581-586 (1993)

6. Voľmir, A.G.: Gibkije plastinky i oboločky. Gosizdat, Moskva (1956) (in Russian) 